\title{
What Have We Learnt from Experimental Renal Transplantation?
}

\author{
K. Brown R.E. Phillips W. Wong \\ MRC Centre for Transplantation, King's College London, School of Medicine at Guy's, King's and \\ St. Thomas' Hospitals, London, UK
}

\section{Key Words}

Kidney transplantation $\cdot$ Immunology $\cdot$ Experimental animal

\begin{abstract}
The first series of kidney transplantation performed in the experimental setting over a century ago and its subsequent translation into humans has initiated a whole new facet of medical practice that has benefited a large number of patients with end-stage kidney and other organ failure. It has proven to be an indispensable tool in our quest to advance our skills and knowledge and continues to play a role in the development of better treatment protocols. Here, we discuss the advantages and drawbacks of this technique and use key examples to illustrate how it has been exploited to achieve our goals.

Copyright $\odot 2010$ S. Karger AG, Basel
\end{abstract}

\section{Introduction and Historical Developments}

Kidney transplantation is the best form of treatment for patients with end-stage renal failure. Today, this is a routine procedure with many units achieving a 1-year graft survival rate of over $90 \%$. Without doubt, this would not have been possible without the skills and knowledge obtained in the past century through experimental renal transplantation. This technique will continue to be an indispensable tool to improve graft outcome and to further understand the complex immunological as well as non-immunological mechanisms involved in clinical kidney transplantation.

The first challenge for kidney transplantation is the technical aspect of the surgical procedure. The donor artery and vein need to be anastomosed to suitable vessels of the recipient. This was first developed by Carrel $[1,2]$ in 1902, and Ullmann [3] went on to perform the first successful kidney transplant in dogs, establishing the basic surgical principles of kidney transplantation. These developments subsequently led to the first series of successful kidney transplants in humans, mainly between identical twins where rejection, the next main hurdle, does not occur.

This next hurdle, rejection, not initially anticipated, was demonstrated by Dempster [4] and Simonsen et al. [5] who transplanted kidneys in dogs and found mononucleocytes around the blood vessels and glomeruli. It was also experimental kidney transplantation that provided the first practical solution to overcome rejection. Again, using a dog kidney transplant model, Calne [6] demonstrated that 6-mercaptopurine (subsequently de-

\section{KARGER \\ Fax +4161306 1234 \\ E-Mail karger@karger.ch}

www.karger.com
(C) 2010 S. Karger AG, Basel

$1660-2129 / 10 / 1151-0009 \$ 26.00 / 0$

Accessible online at:

www.karger.com/nee
Dr. Wilson Wong

MRC Centre for Transplantation, Guy's Hospital

5th Floor, Tower Wing

London SE1 9RT (UK)

Tel. +44 207188 1522, Fax +44 207188 5660, E-Mail wilson.wong@ kcl.ac.uk 
veloped into azathioprine) was able to prevent rejection, enabling successful kidney transplantation to be performed outside the context of identical twins. Following this initial success, other key immunosuppressive agents used today in the clinic such as calcinuerin inhibitors and mTOR inhibitors were also developed in animal models.

Today, experimental kidney transplantation falls into 2 mainstreams: large and small animal models, each with their own advantages and disadvantages, complementing each other to enable investigators to advance our knowledge of transplantation science.

\section{Small Animal Models}

The development of experimental kidney transplantation in rats and mice in the 1960s and 1970s $[7,8]$ has accelerated our understanding of transplantation immunobiology as well as providing a platform for the development of novel therapies to prevent graft rejection. Instead of transplanting tumors or skin grafts, a clinically relevant, vascularized model can be used. Unlike transplanting skin or heart grafts, the function of the transplanted kidney can be measured objectively by monitoring plasma blood urea nitrogen or creatinine. More sophisticated techniques such as inulin clearance or 24-hour urinary protein leak can also be performed to provide more information on graft function. Histological examination of the transplanted kidney is also a fertile ground for investigation as most investigators are proficient in examining histological sections from their clinical practice. In addition, modern molecular biology techniques can provide information on detailed molecular events within the grafts.

A major drawback of using rodents is that the operation itself is technically challenging, due to their small size. Nonetheless, kidney transplantation in rat is now a widely used technique, although mouse kidney transplantation can only be successfully performed in a few centres around the world. In the experience of the authors, the commonest cause of technical failure is due to poor or non-perfusion of the donor kidney. This is because the donor aortic patch used is much smaller than the ascending aortic arch, which is used for heterotopic cardiac transplantation. As a result, even if the anastomosis is done well, the diameter of the lumen at the site of the anastomosis may not be large enough to allow sufficient blood through. Careful hydration of the recipient and use of inhalation anaesthetic, which gives greater and more rapid control of the depth of anaesthesis (and there- fore, blood pressure) will maximize the chance of success. Some investigators have found joining the donor bladder dome to that of the recipient difficult. We prefer to use the Leadbetterr-Politano technique which is much faster with an extremely low technical failure rate.

The single most important feature of small animal models of kidney transplantation is that experiments that are impractical or unethical to perform in humans or larger animals can easily be carried out. The relative low cost and the high volume of turnover, enable statistically powerful studies to be performed in a relatively short period of time. In addition, the well-defined major histocompatibility complex (MHC) of rodents, the availability of a large variety of inbred strains, as well as congenic, mutant, transgenic, knockout and knockin animals (aided by their short gestation period to enable rapid backcrossing), together with the tools such as monoclonal antibodies, all contribute to the merit of this model. The entire genome of a commonly used strain, C57BL/6 has been sequenced in its entirety, with 17 more standard strains to follow. As a result, there have been numerous studies based on rodent kidney transplants. In this review, we do not aim to be comprehensive; instead, we will discuss some seminal studies that take advantage of these key features.

\section{Classical Experiments in Cellular Transplant Immunology}

The factors discussed above have enabled many basic and widely applicable elements of the immune response against the graft to be established. For example, the importance of passenger dendritic leukocytes in the alloimmune response was demonstrated a series of classical experiments [9-11]. Passenger leukocytes of $(A S \times A U G) F_{1}$ rat kidneys were replaced with parental AS type by transplanting (parking) them in AS recipients given AS antiAUG strain antiserum to prevent rejection. When subsequently transplanted into secondary naïve AS recipients, these kidneys were not rejected. Rejection was restored upon injection of donor strain dendritic cells. The ability to breed $\mathrm{F}_{1}$ animals from inbred strains was essential for the success of these experiments.

\section{Use of Genetically Modified Animals}

The generation of genetically modified animals, e.g. knockouts, has allowed individual components of the immune system to be studied. For example, the importance of the complement cascade in the immune response against the graft was demonstrated by the transplantation of $\mathrm{C}^{--}$mouse kidneys, which have greatly pro- 
longed survival in allogeneic recipients compared to kidneys from wild type animals [12]. This study and others have not only allowed a greater understanding of the alloimmune response, but have also prompted the development of novel therapeutic agents for use in transplantation. This field is likely to grow along with the availability of new genetically modified animals.

\section{Testing Novel Therapies}

The relative low cost and high turnover has enabled a large number of novel therapies to be tested in a rapid fashion to select promising agents for more expensive large animal and eventually human studies. For example, many studies in animals showed the importance of costimulation for full activation of $\mathrm{T}$ cells, and also that blockade of co-stimulatory pathways can prolong graft survival. CTLA-4Ig, which blocks the CD28-CD80/86 pathway, was demonstrated to prevent acute rejection in a rat kidney transplant model, and chronic rejection in a mouse renal allograft model $[13,14]$. Based on these rodent findings, CTLA-4Ig has been tested in a non-human primate model, and an analogue of CTLA-4Ig (Belatacept) has completed phase II clinical trials in humans for renal transplantation [15] and is now in clinical use. Experimental kidney transplantation in this case was vital for both the understanding of the importance of the costimulatory pathways, and for the development and testing of the CTLA-4Ig fusion protein. It would simply be impractical and unethical not to use small animals first to select promising agents for pre-clinical and clinical studies.

\section{Investigation of Clinical Phenomena}

Experimental kidney transplantation can also be used, not only as a starting point for novel therapies, but also to explain results obtained in the clinic using such therapies. The monoclonal antibody alemtuzumab (Campath$1 \mathrm{H}$ ) depletes $\mathrm{T}$ and $\mathrm{B}$ cells after transplantation, but a high rate of rejection still occurs $[16,17]$. Experiments in animal models have revealed a possible reason for this. Cells that escape depletion undergo homeostatic proliferation in a lymphopaenic environment. Our laboratory [18] and others [19-21] have shown that following homeostatic proliferation, $T$ cells acquire a memory phenotype and have augmented ability to reject kidney allografts as well as being resistant to tolerance induction, offering an explanation for the need to use conventional immunosuppressants in conjunction with alemtuzumab.

Experimental Kidney Transplantation

\section{Avoiding Ethical Difficulties}

The study of kidney tissue in human recipients that are tolerant to their kidney grafts has been extremely difficult, mainly because it is a rare event, but also as it would be unethical to biopsy kidney grafts in patients with stable function that are off immunosuppression. To address this, we have used a mouse model of spontaneous tolerance to study the infiltration of regulatory $\mathrm{T}$ cells in tolerant allografts and demonstrated that mononuclear cells infiltrated tubules in both rejecting and tolerant grafts. However, tolerant kidney grafts demonstrated a higher and sustained level of Foxp $3^{+}$regulatory cells. Importantly, a significant proportion of these cells were found within tubules. In cases where graft function was normal, these cells were not harmful to the kidney and could be said to be mimicking, rather than causing, rejection [22].

\section{A Tool to Study Immunological Phenomenon}

Apart from its obvious application in the investigation of transplantation, experimental kidney transplantation has also been used to investigate basic immunological phenomena. By transplanting MHC-mismatched kidneys, one is in effect creating a chimera which may have unforeseen immunological effects at the cellular level. It has been known for some time that when 2 different cell populations are mixed together in vitro, they can exchange their cell surface molecules, including MHC molecules. Whether this is also a naturally occurring physiological phenomenon in vivo or purely an in vitro artefact is not known. Kidney transplantation is ideal to study this as the mismatched MHC molecules from the donor can be easily tracked by monoclonal antibodies. Using this technique, our laboratory has demonstrated that there is extensive and bi-directional MHC transfer between donor and recipient cells following kidney transplantation. This finding may have important implications on the priming of the alloimmune response, as well as in other fields of immunobiology such as vaccination, tumour immunology and autoimmunity [23].

\section{Shortcomings of Small Animal Experiments}

Although small animal models represent useful tools to study transplantation, they are not a perfect reflection of the clinical situation. This is illustrated by the ease of inducing transplant tolerance in small animals, and if this were an accurate representation of the human situation, tolerance would have been achieved many years ago in the clinic. There are many possible reasons for this. Firstly, most experiments are conducted using inbred mice which are thought to be less likely to reject transplanted organs.

Nephron Exp Nephrol 2010;115:e9-e14 
Secondly, although donor and recipient strains used are often said to be fully allogeneic, the MHC molecules in terms of the amino acid sequences are still very similar between strains. This is not the case in humans as human leucocyte antigen molecules are highly polymorphic. Thirdly, in humans, during rejection, the majority of $\mathrm{T}$ cells involved in the alloresponse are likely to be memory $\mathrm{T}$ cells developed from previous encounters with micro-organisms, a phenomenon known as heterologous immunity [24]. Experimental animals that are kept in highly controlled environments with limited exposure to environmental pathogens are unlikely to have strong heterologous immunity. The life span of small animals is much shorter than that of humans. Inbred laboratory mice rarely live beyond 2 years of age even without undergoing any experimental procedures. This puts into question the relevance of such models to chronic pathology such as interstitial fibrosis and tubular atrophy, even though there are numerous models available for this purpose, such as one developed by us [18].

In addition, there are important differences between the physiology (such as resistance to calcinuerin toxicity) and immune system of small animals and humans which makes it necessary to use large animal models, especially non-human primates, to test theories and therapies developed in small animals.

\section{Large Animal Models}

\section{Pigs and Non-Human Primates}

Today, experimental kidney transplantation in large animal models is mostly carried out in pigs and non-human primates. Pigs are ethically more acceptable than non-human primates as an experimental model and inbred pigs with well-defined MHC have been established and characterized specifically to facilitate transplant research [25]. They have shorter life cycles than primates and can produce large litters. More importantly, genetically modified herds such as those expressing complement regulatory proteins for xenotransplantation can be created within a fairly short period of time. Their size is similar to that of humans and for this reason they are the preferred donors in xenotransplantation.

Non-human primates are also commonly used in experimental kidney transplantation, especially as recipients of xenografts. Macaques are thought to be the most useful as they are small and exhibit cross-reactivity with a large number of human immune molecules allowing assessment of therapies using monoclonal antibodies toward human targets.

\section{Translation into Clinical Use}

In contrast to small animal models, which have been critical in defining fundamental biological mechanisms in kidney transplantation, translation of experimental immunosuppression and tolerance induction protocols into the human setting relies heavily on large animal models. Many novel treatments have been validated and toxicity tested in at least one large animal model before they progress to human clinical trials. Essentially, there are 2 reasons for this. Firstly, large animal physiology more closely resembles that of human and the likely efficacy of a treatment in human transplantation is often inferred by its performance in a large animal model. An early and pivotal example of this was the successful use of 6-mercaptopurine as mentioned above [6]. In fact, all the commonly used immunosuppressants were first evaluated in large animal models, as were more recent strategies such as co-stimulatory blockade, T cell depletion and induction of hematopoietic chimerism. Secondly, in addition to assessing efficacy, large animal models are also important in determining the safety of a given treatment. The importance of large animal models is shown by the number of therapies which fail at this stage of testing.

A recent example of the translation from animal models to the clinic is the work of Sachs and colleagues [25] to create mixed chimerism in transplant recipients without the need for lethal irradiation. Mixed chimerism had previously been shown to be beneficial to the acceptance of allografts but the whole body irradiation required to induce it was too toxic to use in the clinic. Using rodents it was possible to develop a method of achieving mixed chimerism without lethal irradiation. It was then tested in a miniature swine model, where tolerance to kidney allografts was induced. This technique has since been used successfully by the same group in human renal transplant recipients, and provides an exciting prospect for achieving the aim of transplant tolerance in the clinic [26].

It is clear, however, that large animal models can be imperfect in predicting both safety and efficacy. Through experience, we know that humans are still more resistant to tolerance than their large animal counterparts for reasons that are not entirely clear. Furthermore, drug metabolism and absorption can differ significantly between humans and large animal models, meaning drug responses and toxicity profiles are often poorly predictive. Despite these shortcomings, large animal models remain the best way for us to assess safety and efficacy before phase I human clinical trials.

An example of the difficulties in translating results from animal studies into the clinic can be seen in the de- 
velopment of an anti-CD154 antibody, which again blocks a co-stimulation pathway. Antibodies were first tested with great success in small animal models, and then with similar results in non-human primates [27]. However, clinical trials had to be discontinued due to thrombotic episodes not seen in animal models.

\section{Xenotransplantation Research}

The shortage of donor organs available for human recipients fuels interest in using organs from animals. Research into xenotransplantation usually involves using pigs as donors and non-human primates as recipients. Old World non-human primates, like humans, but unlike other species including pigs, lack the galactosyl- $\alpha 1,3$ galactose molecule responsible for causing hyperacute rejection. They are, therefore, the obvious choice as recipients. Yamada et al. [28] have achieved survival of up to 80 days of alpha-1,3-galactosyltransferase knockout pig kidneys in baboons; however, this required profound immunosuppression including T cell depletion, CD154 blockade and mycophenolate mofetil, in combination with splenectomy and thymectomy of the recipient and co-transplant of the donor thymus. Much progress has been made in this field, but many other challenges remain before xenotransplantation can be applied in the clinic.

\section{Limitations of Large Animal Models}

There are several challenges in the use of large animals in experimental kidney transplantation. There is more resistance against use of larger animals such as non-hu- man primates and dogs compared to smaller animals such as rodents. The ethics of large animal experimentation has thus evolved to a similar complexity as that for humans, in that experiments must all be based on prior in vitro observations, and often in vivo evaluation in smaller animals. This means far more preliminary work must be performed. This is not necessarily a disadvantage, as large animal experiments are particularly expensive and it probably would not be cost effective to undertake experiments which had not been assessed in less costly small animal models. Additionally, with the exception of a small number of established inbred pig herds, most large animals are outbred, making replication of experiments more difficult. It takes longer to genetically modify them due to their longer life cycles compared with rodents. Finally, there are less available immunological reagents such as monoclonal antibodies for the larger species making experiments more challenging.

\section{Conclusions}

We owe much of what we know now and what we can do to experimental kidney transplantation. From the early pioneers to today's cutting edge researchers, all have added invaluable information to benefit recipients of kidney grafts. There is no doubt that this technique will have a pivotal role to play in the future, in areas such as stem cell research, cloning and in overcoming the major hurdles in transplantation - xenotransplantation, tolerance and chronic rejection.

\section{References}

1 Carrel A: Anastomose bout a bout de la jugulaire et de la carotide primitive. Lyon Med 1902;99:114.

2 Carrel A: Presentation d'un chien porteur d'une anastomose arterioveineuse. Lyon Med 1902;99:152.

3 Ullmann E: Experimentelle Nierentransplantation. Wien Klin Wochenschr 1902;11: 281-285.

4 Dempster WJ: Kidney homotransplantation. Br J Surg 1953;40:447-465.

5 Simonsen M, Buemann J, Gammeltoft A, Jensen F, Jorgensen K: Biological incompatibility in kidney transplantation in dogs. I. Experimental and morphological investigations. Acta Pathol Microbiol Scand 1953;32: $1-35$.
6 Calne RY: The rejection of renal homografts. Inhibition in dogs by 6-mercaptopurine. Lancet 1960;1:417-418.

7 Miller BF, Gonzalez E, Wilchins LJ, Nathan P: Kidney transplantation in the rat. Nature 1962;194:309-310.

-8 Russell PS, Chase CM, Colvin RB, Plate JM: Kidney transplants in mice. An analysis of the immune status of mice bearing longterm, H-2 incompatible transplants. J Exp Med 1978;147:1449-1468.

-9 Batchelor JR, Welsh KI, Maynard A, Burgos $\mathrm{H}$ : Failure of long surviving, passively enhanced kidney allografts to provoke T-dependent alloimmunity. I. Retransplantation of $(\mathrm{AS} \times \mathrm{AUG}) \mathrm{F}_{1}$ kidneys into secondary AS recipients. J Exp Med 1979;150:455-464.
10 Welsh KI, Batchelor JR, Maynard A, Burgos $\mathrm{H}$ : Failure of long surviving, passively enhanced kidney allografts to provoke T-dependent alloimmunity. II. Retransplantation of $(\mathrm{AS} \times \mathrm{AUG}) \mathrm{F}_{1}$ kidneys from AS primary recipients into $(\mathrm{AS} \times \mathrm{WF}) \mathrm{F}_{1}$ secondary hosts. J Exp Med. 1979;150:465470.

11 Lechler RI, Batchelor JR: Restoration of immunogenicity to passenger cell-depleted kidney allografts by the addition of donor strain dendritic cells. J Exp Med 1982;155: 31-41.

12 Pratt JR, Basheer SA, Sacks SH: Local synthesis of complement component $\mathrm{C} 3$ regulates acute renal transplant rejection. Nat Med 2002;8:582-587. 
-13 Harada H, Ishikura H, Nakagawa I, Shindou J, Murakami M, Uede T, et al: Abortive alloantigen presentation by donor dendritic cells leads to donor-specific tolerance: a study with a preoperative CTLA4lg inoculation. Urol Res 2000;28:69-74.

- 14 Azuma H, Chandraker A, Nadeau K, Hancock WW, Carpenter CB, Tilney NL, et al: Blockade of T-cell costimulation prevents development of experimental chronic renal allograft rejection. Proc Natl Acad Sci USA 1996;93:12439-12444.

-15 Vincenti F, Larsen C, Durrbach A, Wekerle T, Nashan B, Blancho G, et al: Costimulation blockade with Belatacept in renal transplantation. N Engl J Med 2005;353:770-781.

-16 Kirk AD, Hale DA, Mannon RB, Kleiner DE, Hoffmann SC, Kampen RL, et al: Results from a human renal allograft tolerance trial evaluating the humanized CD52-specific monoclonal antibody alemtuzumab (CAMPATH-1H). Transplantation 2003;76:120129.

-17 Knechtle SJ, Pirsch JD, H. Fechner J J, Becker BN, Friedl A, Colvin RB, et al: Campath-1H induction plus rapamycin monotherapy for renal transplantation: results of a pilot study. Am J Transplant 2003;3:722-730.
18 Moxham VF, Karegli J, Phillips RE, Brown KL, Tapmeier TT, Hangartner R, et al: Homeostatic proliferation of lymphocytes results in augmented memory-like function and accelerated allograft rejection. J Immunol 2008;180:3910-3918.

19 Cho BK, Rao VP, Ge Q, Eisen HN, Chen J: Homeostasis-stimulated proliferation drives naive $\mathrm{T}$ cells to differentiate directly into memory T cells. J Exp Med 2000;192:549 556.

20 Murali-Krishna K, Ahmed R: Cutting edge: naive $\mathrm{T}$ cells masquerading as memory cells. J Immunol; 2000;165:1733-1737.

21 Gudmundsdottir H, Turka LA: A closer look at homeostatic proliferation of CD4+ T cells: costimulatory requirements and role in memory formation. J Immunol 2001;167: 3699-3707.

22 Brown K, Moxham V, Karegli J, Phillips R, Sacks SH, Wong W: Ultra-localization of Foxp3+ T cells within renal allografts shows infiltration of tubules mimicking rejection. Am J Pathol 2007;171:1915-1922.

23 Brown K, Sacks SH, Wong W: Extensive and bidirectional transfer of major histocompatibility complex class II molecules between donor and recipient cells in vivo following solid organ transplantation. FASEB J 2008; $22: 3776-3784$.
24 Adams AB, Williams MA, Jones TR, Shirasugi N, Durham MM, Kaech SM, et al: Heterologous immunity provides a potent barrier to transplantation tolerance. J Clin Invest 2003;111:1887-1895.

-25 Mezrich JD, Haller GW, Arn JS, Houser SL, Madsen JC, Sachs DH: Histocompatible miniature swine: an inbred large-animal model. Transplantation 2003;75:904-907.

26 Fuchimoto Y, Huang CA, Yamada K, Shimizu A, Kitamura H, Colvin RB, et al: Mixed chimerism and tolerance without whole body irradiation in a large animal model. J Clin Invest 2000;105:1779-1789.

27 Kirk AD, Burkly LC, Batty DS, Baumgartner RE, Berning JD, Buchanan K, et al: Treatment with humanized monoclonal antibody against CD154 prevents acute renal allograft rejection in nonhuman primates. Nat Med 1999;5:686-693.

28 Yamada K, Yazawa K, Shimizu A, Iwanaga T, Hisashi Y, Nuhn M, et al: Marked prolongation of porcine renal xenograft survival in baboons through the use of alpha1,3-galactosyltransferase gene-knockout donors and the cotransplantation of vascularized thymic tissue. Nat Med 2005;11:32-34. 